\title{
Economic evaluation of the sFlt-1/PIGF ratio for the short-term prediction of preeclampsia in a Japanese cohort of the PROGNOSIS Asia study
}

\author{
Akihide Ohkuchi ${ }^{1} \cdot$ Hisashi Masuyama ${ }^{2} \cdot$ Tatsuo Yamamoto $^{3} \cdot$ Takashi Kikuchi $^{4} \cdot$ Naoko Taguchi $^{4} \cdot$ Cyrill Wolf $^{5}$. \\ Shigeru Saito ${ }^{6}$
}

Received: 27 August 2020 / Revised: 19 November 2020 / Accepted: 16 December 2020 / Published online: 16 February 2021

(c) The Author(s) 2021. This article is published with open access

\begin{abstract}
The PRediction of short-term Outcomes in preGNant wOmen with Suspected preeclampsIa Study (PROGNOSIS) Asia validated the use of the soluble fms-like tyrosine 1/placental growth factor (sFlt-1/PlGF) ratio cutoff value of $\leq 38$ to rule out the occurrence of preeclampsia in the short term in Asian women. We assessed the economic impact of the introduction of the sFlt-1/PlGF ratio test for predicting preeclampsia in Japan using data from the Japanese cohort of PROGNOSIS Asia. The cost analysis was developed with estimates in either a no-test scenario, with clinical decisions based on standard diagnostic procedures alone, or a test scenario, in which the sFlt-1/PlGF ratio test was used in addition to standard diagnostic procedures. For both scenarios, rates of hospitalization and other test characteristics were obtained from the results for the Japanese cohort in PROGNOSIS Asia. The total cost per patient was the main outcome of this cost analysis model. Introduction of the sFlt-1/PlGF ratio test using a cutoff value of 38 resulted in a reduced hospitalization rate compared with the rate in the no-test scenario (14.4\% versus $8.7 \%$ ). The reduction in the rate of hospitalizations led to an estimated 16373 JPY reduction in healthcare costs per patient. The sFlt-1/PlGF ratio test is likely to reduce the unnecessary hospitalization of women at low risk of developing preeclampsia in the short term while also identifying high-risk individuals requiring appropriate management. Reducing unnecessary hospitalizations would result in significant cost savings in the Japanese healthcare system.
\end{abstract}

Keywords Cost saving $\cdot$ Prediction $\cdot$ Japan $\cdot$ Preeclampsia $\cdot$ sFlt-1/PlGF ratio

Supplementary information The online version contains supplementary material available at https://doi.org/10.1038/s41440021-00624-2.

Cyrill Wolf

cyrill.wolf@roche.com

Jichi Medical University Hospital, Tochigi, Japan

2 Okayama University, Okayama, Japan

3 Nihon University Itabashi Hospital, Tokyo, Japan

4 Roche Diagnostics K.K., Tokyo, Japan

5 Roche Diagnostics International Ltd, Rotkreuz, Switzerland

6 Toyama University Hospital, Toyama, Japan

\section{Introduction}

Preeclampsia is a hypertensive disorder of pregnancy (HDP), defined as new-onset hypertension and proteinuria or other maternal organ dysfunction after 20 weeks of gestation [1]. Globally, preeclampsia affects $2-8 \%$ of pregnancies $[2,3]$ and $\sim 3 \%$ of singleton pregnancies in Japan [4]. It is associated with substantial maternal morbidity and mortality [5, 6]. In Japan, between 1989 and 2004, 193 cases of maternal deaths were due to direct obstetric causes; of those deaths, 41 (21.2\%) were associated with disseminated intravascular coagulation due to HDP [7]. Preeclampsia also contributes significantly to healthcare costs $[5,8,9]$.

Guidelines from the Japan Society of Obstetrics and Gynecology recommend the hospitalization of women diagnosed with preeclampsia but do not provide clear guidance on the management of women with risk factors only for the 
condition [10]. There is no centralized maternal care system in Japan, and the use of midwives is uncommon. Therefore, women often visit obstetricians in the clinic, which may further impact the management of women with risk factors for preeclampsia. As such, there is an unmet clinical need for objective indicators to predict preeclampsia. Currently, women are referred to perinatal care centers only if they develop severe preeclampsia, hemolysis, elevated liver enzymes, low platelet count (HELLP) syndrome, seizures, or mild/moderate/accidental hypertension.

Preeclampsia is associated with dysregulation of angiogenic factors such as soluble fms-like tyrosine 1 (sFlt-1) and placental growth factor (PIGF) [11-14]. Quantification of the sFlt-1/PIGF ratio, derived from Elecsys ${ }^{\circledR}$ sFlt-1 and Elecsys PIGF immunoassay (Roche Diagnostics, Mannheim, Germany) results, has been shown to be a useful biomarker test for aiding the diagnosis and short-term prediction of preeclampsia [15-22].

The global, multicenter, noninterventional PRediction of short-term Outcomes in preGNant wOmen with Suspected preeclampsIa Study (PROGNOSIS) derived and validated an sFlt-1/PIGF ratio cutoff of 38 to predict the development of preeclampsia in women with clinical suspicion of the condition. The negative predictive value (NPV) of an sFlt-1/PlGF ratio $\leq 38$ to rule out preeclampsia was $99.3 \%$ within 1 week and $94.3 \%$ within 4 weeks; the positive predictive value (PPV) to rule in preeclampsia within 4 weeks was $36.7 \%$ $[17,22,23]$. PROGNOSIS Asia validated the sFlt-1/PIGF ratio cutoff of 38 for short-term preeclampsia prediction in Asian women with suspected preeclampsia [15]. Analysis of PROGNOSIS Asia data demonstrated an NPV of $98.6 \%$ for an sFlt-1/PIGF ratio of $\leq 38$ to rule out preeclampsia within 1 week and an NPV of $95.1 \%$ to rule it out within 4 weeks. The PPV to rule in preeclampsia within 1 week was $17.9 \%$, and the PPV to rule it in within 4 weeks was $30.3 \%$.

The economic impact of implementing the sFlt-1/PIGF ratio test, based on PROGNOSIS data, has been assessed in the UK, Italy, Brazil, Germany, Switzerland, and the US [24-30]. The authors concluded that introducing the test into clinical practice for women with suspected preeclampsia would provide cost savings for the respective healthcare providers, mainly due to the increased ability to rule out preeclampsia and thereby reduce unnecessary hospitalizations. However, this type of economic assessment is specific to the individual patient population and healthcare provider tested and cannot be extrapolated to other healthcare systems. To date, the economic impact of this test has not been assessed using patient-level data from PROGNOSIS Asia. The present analysis thus aimed to evaluate the economic impact of implementing the sFlt-1/ PIGF ratio test for the short-term prediction of preeclampsia in Japanese women with suspected preeclampsia using patient-level data from PROGNOSIS Asia [15, 31].

\section{Methods}

\section{The Japanese cohort from PROGNOSIS Asia}

PROGNOSIS Asia was a prospective, multicenter observational study of 764 women with suspected preeclampsia who were enrolled at 25 sites across Asia between December 2014 and December 2016 [15]. The Japanese cohort in PROGNOSIS Asia included 192 enrolled women at eight sites in Japan between June 2015 and May 2016. The key inclusion criteria for the Japanese cohort were being a pregnant woman $\geq 18$ years of age who presented with suspected preeclampsia from 18 weeks +0 days gestation to 36 weeks +6 days gestation. The inclusion criteria were adapted to align with local practice guidelines as required; the criterion relating to the lower limit of blood pressure was adapted to reflect Japanese practice, and the lower limit of the eligible gestational age range was adapted to 18 weeks (rather than 20 weeks for the other Asian countries). The key exclusion criteria were manifest preeclampsia or confirmed diagnosis of HELLP syndrome; multiple pregnancies; confirmed diagnosis of a fetal chromosomal abnormality; or treatment with an investigational medicine within 90 days. The study protocol was approved by local ethics committees and institutional review boards at each of the eight Japanese sites involved prior to study initiation, and all participants provided written informed consent. The study was conducted in accordance with the Declaration of Helsinki and International Conference on Harmonisation guidelines for Good Clinical Practice.

Patient-level data from the Japanese cohort in PROGNOSIS Asia were used to determine the following factors in the present economic model: (a) the proportion of women hospitalized with suspected preeclampsia in current practice (i.e., in the absence of sFlt-1/PlGF ratio results); (b) the correlation between hospitalization and the sFlt-1/PIGF ratio (where values $\leq 38$ suggest low risk and $>38$ suggest high risk [22]); and (c) the relationship among the sFlt-1/PIGF ratio, hospitalization, and a confirmed diagnosis of preeclampsia. The study was also used to provide information on the length of hospital stay before and after preeclampsia onset and for women who did not develop preeclampsia.

\section{Cost analysis model}

For this cost analysis, an economic model was developed to estimate direct medical costs associated with the diagnosis and management of women with suspected preeclampsia from a Japanese healthcare system perspective. The model simulated the progression of a cohort of Japanese women through a treatment pathway from the first presentation with 
clinical suspicion of preeclampsia to the point of delivery, which was determined by the assessed risk of developing preeclampsia and the consequent decision of whether to hospitalize the patient or manage in an outpatient setting. The model also included the cost of neonatal intensive care unit (NICU) admission and treatment of neonates with respiratory distress syndrome (RDS) born to mothers with suspected preeclampsia. The model assumed that every neonate born with RDS would be admitted to the NICU; that the rate of RDS in neonates from hospitalized mothers with suspected preeclampsia is $12 \%$; and that hospitalizing women with suspected preeclampsia based on the sFlt-1/ PIGF ratio result and corticosteroid administration, would reduce the RDS rate by $20 \%$ [32]. The calculation of a $20 \%$ reduction was derived from PreOS, a multicenter, noninterventional study run in Germany and Austria evaluating the sFlt-1/PIGF ratio test for its use in diagnosis and clinical decision making [33]. The study report showed that the overall reduction in the RDS rate in PreOS was $25.6 \%$ [unpublished data]; however, the sFlt-1/PIGF ratio cutoff utilized to rule out preeclampsia in PreOS was 33, whereas a higher cutoff of 38 was used in PROGNOSIS Asia. Therefore, we assumed that the reduction in the RDS rate would decrease from 25.6 to $20 \%$.

The model assumptions were estimated based on values from the Neonatal Research Network of Japan database [32]. The incremental value of implementing the sFlt-1/ PIGF ratio test was evaluated by comparing expected management costs in two scenarios. In the no-test scenario, diagnosis and management of women with suspected preeclampsia were performed according to current standard-ofcare procedures; the hospitalization rate was based on the observed rate in the Japanese cohort in PROGNOSIS Asia. In the test scenario, diagnosis and management of women with suspected preeclampsia were performed according to current standard-of-care procedures plus the results of the sFlt-1/PlGF ratio test. The results of the sFlt-1/PlGF ratio test enabled classification of the women as either low $(\leq 38)$ or high (>38) risk for developing preeclampsia. The proportion of women in each group was based on the observed proportions in the Japanese cohort in PROGNOSIS Asia. Notably, in PROGNOSIS Asia, the results of the sFlt-1/ PIGF ratio test were blinded to the clinicians to ensure that clinical decision making was not impacted by the results. In the low-risk group, the hospitalization rate $(0.56 \%)$ was based on the assumption that women with an sFlt-1/PlGF ratio $\leq 38$ (with an NPV to rule out PE within 1 week of $98.6 \%$ and a value of $95.1 \%$ to rule out within 4 weeks [15]) would be hospitalized if their blood pressure was $\geq 160 / 110$ $\mathrm{mmHg}$ (as per the Best Practice Guide 2015 for Care and Treatment of Hypertension in Pregnancy [34]). In the highrisk group, the model used a conservative estimate for the hospitalization rate (40\%) based on the observed rate in the
Japanese cohort in PROGNOSIS Asia, where the hospitalization rate was $27 \%$.

The patient pathway was based on the PROGNOSIS Asia study and adapted to Japanese patient management procedures; the model assumed that patients not admitted to the hospital were managed in an outpatient setting. The incidence of preeclampsia was assumed to be unaffected by the introduction of the sFlt-1/PIGF ratio test. No direct information was available from the PROGNOSIS study to determine what clinical decisions might have been made if physicians had been given access to sFlt-1/PIGF ratio results. A clinical algorithm was developed to estimate the disposition of women according to their sFlt-1/PIGF ratio.

\section{The model cohort}

A model cohort of 31,000 women (based on estimates of pregnant women with preeclampsia in Japan at any given time) with suspected preeclampsia and the Japanese-specific cohort from PROGNOSIS Asia were used to compare the no-test and test scenarios.

\section{Costs used in the analysis}

The expected costs of caring for women with suspected preeclampsia and neonates with RDS used in the modeling were based on annual data from the Ministry of Health, Labor \& Welfare report (Table 1). The model assumes that all patients treated with high-intensity management are prescribed oral corticosteroids to reduce the risk of complications at a cost of 1752 Japanese yen (JPY) per patient. The cost of the sFlt-1/PIGF ratio test was estimated to be $9000 \mathrm{JPY}$. Outpatient costs were calculated for every woman in the

Table 1 Expected maternal and neonatal care costs used in the economic modelling (based on annual data from Ministry of Health, Labour \& Welfare reports [37-39])

\begin{tabular}{ll}
\hline Cost type & Cost, JPY \\
\hline Maternal care & \\
Outpatient $^{\mathrm{a}}$ & 57,053 \\
Hospitalization (per day) & 87,300 \\
sFlt-1/PlGF ratio test & 9000 \\
Neonatal care & \\
Maternal corticosteroid treatment & 1752 \\
NICU stay (per day) & 101,302 \\
\hline
\end{tabular}

$J P Y$ Japanese yen, NICU neonatal intensive care unit, $P l G F$ placental growth factor, $s F l t-1$ soluble fms-like tyrosine kinase-1

${ }^{a}$ Outpatient costs were calculated for every woman entering the model, including those who were hospitalized, as it was assumed that hospitalized women would also be treated in an outpatient setting at some stage 
model, including hospitalized women, who were assumed to be treated in an outpatient setting at some stage.

\section{Sensitivity analyses}

Sensitivity analyses were conducted to test the robustness of our results for a range of scenarios in which key model parameters were altered. The analysis included (a) an increase in the hospitalization rate in the no-test scenario to the overall hospitalization rate in PROGNOSIS Asia; (b) variations in the cost of the sFlt-1/PlGF ratio test, whereby the cost was increased and reduced by $20 \%$; (c) a second sFlt-1/PlGF ratio test for every woman (also an estimated cost of $9000 \mathrm{JPY}$ ); (d) an increase in the hospitalization rate for women with sFlt-1/PlGF ratios $\leq 38$ to $4 \%$; (e) a decrease in the hospitalization rate for women with sFlt-1/PIGF ratios $\leq 38-0 \%$; and (f) an increase of 10 percentage points in the hospitalization rate for women with sFlt-1/PlGF ratios $>38$.

\section{Results}

\section{Preeclampsia-related hospitalization rates}

Data for 180 Japanese women (out of 192 women enrolled) with suspected preeclampsia from PROGNOSIS Asia were eligible for the analysis. In the no-test scenario, $14.4 \%$ of women were hospitalized, and $85.6 \%$ were treated in an outpatient setting (Fig. 1a). The hospitalization rate for this cohort was lower than the overall rate for the entire PROGNOSIS Asia population, in which $26.9 \%$ of women were hospitalized. The average length of hospital stay was 10.4 days in the no-test scenario and 10.2 days in the test scenario (Supplementary Table 1; unpublished data). The overall number of inpatient days included women admitted before preeclampsia (average 4 days); the length of stay for those admitted for the birth period varied depending on whether the women developed preeclampsia (13.6 days) or not (9.3 days). A larger number of women were admitted before the onset of preeclampsia in the no-test scenario $(n=4476)$ than in the test scenario $(n=2691)$. This model assumes that the incidence of preeclampsia is independent of sFlt-1/PlGF ratio testing; as such, the number of birth episodes both with and without preeclampsia remained the same between the test and no-test scenarios.

In the test scenario, $79.4 \%$ of women had sFlt-1/PlGF ratios $\leq 38$, and $0.56 \%$ of them were hospitalized. The remainder of the women $(20.6 \%)$ had sFlt-1/PlGF ratios $>38$, and $40 \%$ of them were hospitalized (Fig. 1b). The hospitalization rate for PROGNOSIS Asia was $27 \%$ in this cohort, but a more conservative rate of $40 \%$ was used for the present analysis. This resulted in an overall hospitalization rate of $8.7 \%$ in the test scenario.
Of the women who were hospitalized in the test scenario, $41.4 \%$ developed preeclampsia in the hospital. By contrast, the rate of women who developed preeclampsia while in the hospital in the no-test scenario was $24.1 \%$. These results support the hypothesis that the test facilitates the appropriate identification and management (i.e., through hospital admission) of women at higher risk of developing preeclampsia, while women at lower risk may be managed in an outpatient setting.

\section{NICU admission rates for RDS}

Applying a 12\% RDS rate (for newborns from hospitalized mothers with signs and symptoms of preeclampsia) and a $20 \%$ reduction in the $\mathrm{RDS}$ rate (if the test is used) to a model cohort of 31,000 women (assuming that the full model cohort of women with suspected preeclampsia would have access to the test) would result in 537.17 NICU admissions in the no-test scenario and 429.73 NICU admissions in the test scenario (Supplementary Table 2).

\section{Base-case cost saving and sensitivity analyses}

Based on the expected maternal and neonatal care costs shown in Table 1, introduction of the sFlt-1/PlGF ratio test is expected to result in cost savings of 16,373 JPY per patient compared with costs in the no-test scenario (Table 2; Supplementary Table 2). These cost savings are largely due to the reduction in hospitalization. To perform the sensitivity analyses, cost savings of 16,373 JPY per patient were used as the base case. The sensitivity analyses supported the robustness of the value of investigating the sFlt-1/PlGF ratio in reducing costs, as the cost savings across all scenarios ranged from 6782 to 69,482 JPY (Table 2; Supplementary Fig. 1).

\section{Discussion}

Our economic analysis of patient-level data from PROGNOSIS Asia shows that implementation of the sFlt-1/PlGF ratio as a diagnostic aid in current standard-of-care procedures could reduce the hospitalization rate for Japanese women with suspected preeclampsia from 14.4 to $8.7 \%$. Notably, the introduction of the sFlt-1/PlGF ratio test would decrease the number of NICU admissions of neonates with RDS born to these women and the associated costs. Our findings suggest that implementation of the sFlt-1/PlGF ratio in routine clinical practice may enable better stratification of women at low-and high-risk of developing preeclampsia and thus facilitate appropriate clinical decision making. Management decisions that include the results of 
A

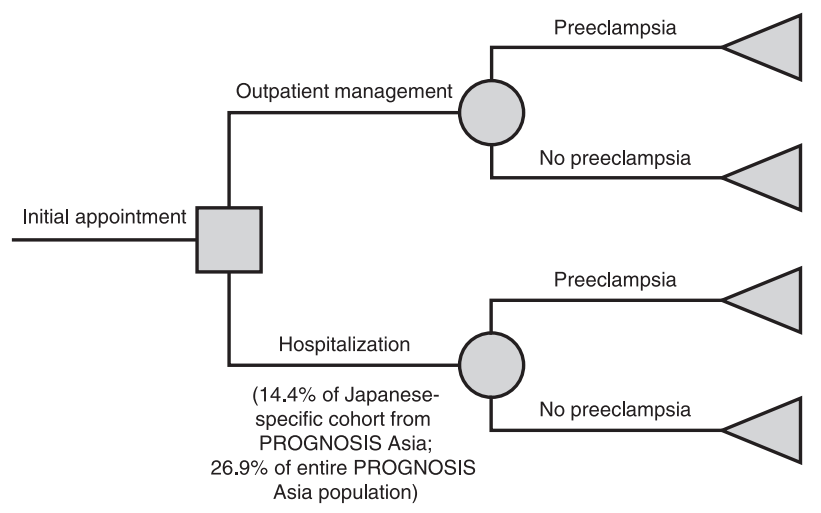

B

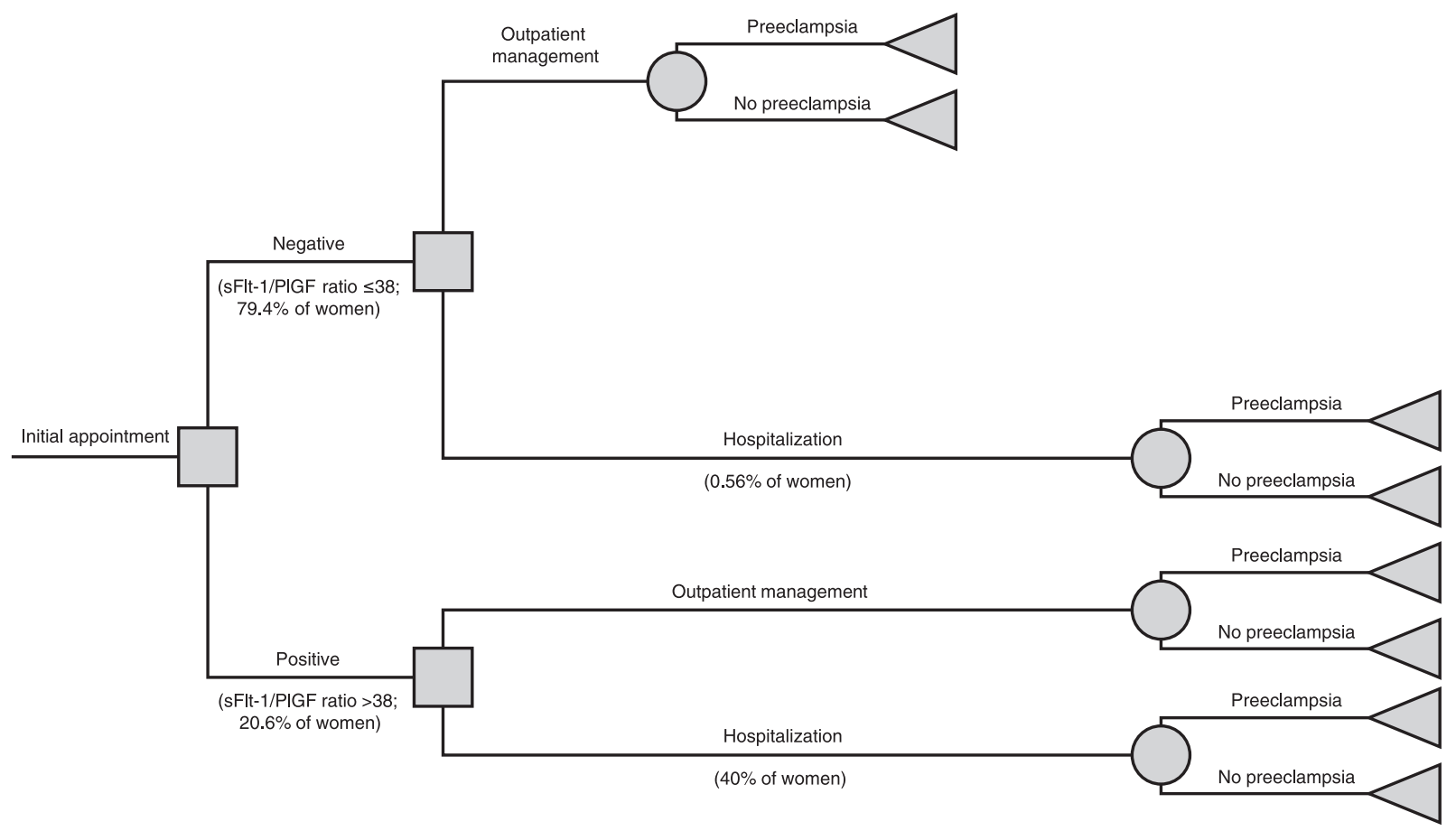

Fig. 1 Decision trees in the (A) no-test scenario (i.e., current standard of care) and (B) test scenario (i.e., current standard of care plus the sFlt-1/PIGF ratio test). PlGF, placental growth factor; PROGNOSIS,

the sFlt-1/PlGF ratio correlated with preeclampsia outcomes better than current diagnostic procedures alone. For patients at low risk for preeclampsia, the reduction in unnecessary hospitalizations and extended monitoring likely improves the cost-effectiveness for healthcare providers in Japan.

In the base case scenario, the decreasing rates of hospitalization and NICU admission due to the introduction of the sFlt-1/PlGF ratio test were associated with cost savings of 16,373 JPY per patient compared with the costs using current standard-of-care procedures alone. The additional cost of the sFlt-1/PIGF ratio test is more than offset by a reduction in hospital resource use. The exact reduction in
PRediction of short-term Outcomes in preGNant wOmen with Suspected preeclampsIa Study; $s F l t-1$, soluble fms-like tyrosine kinase-1

the hospitalization rate and the associated cost savings are dependent on local factors; however, our sensitivity analyses demonstrate that our base case cost assumptions are robust to changes in key parameters in the modeling, including running the test twice for each woman.

Assuming that there are 31,000 pregnant women with suspected preeclampsia in Japan at any given time, the budget impact of including the sFlt-1/PIGF ratio test in routine clinical practice could be 507,560,536 JPY in total savings for the Japanese healthcare system. Prior to implementation by healthcare providers, the costeffectiveness of any new test must be demonstrated in 


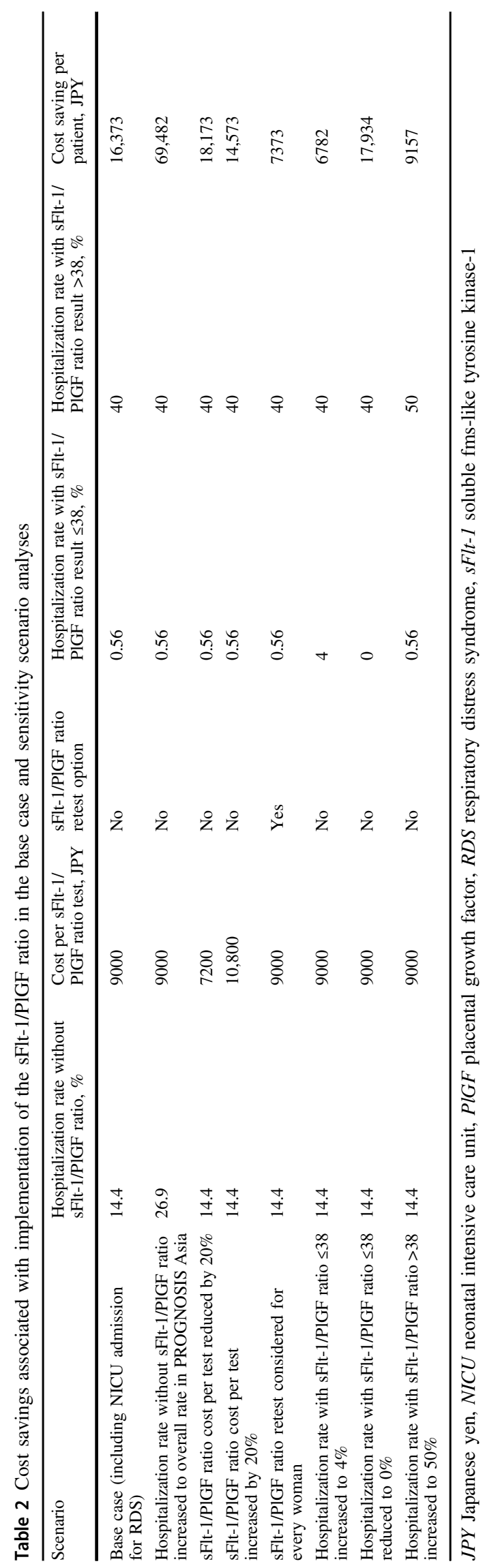

addition to its clinical performance. Similar research on the cost analysis of introducing the sFlt-1/PlGF ratio test has been performed using population-level data from PROGNOSIS in the UK, Italy, Germany, Brazil, Switzerland, and the US [24-30]. In PROGNOSIS, the sFlt-1/PIGF ratio has been shown to improve the short-term prediction of preeclampsia in women with clinical suspicion of the condition, and NICE guidelines recommend the use of the sFlt-1/ PlGF ratio test to rule out preeclampsia, in addition to standard care, in women between 20 and 34 weeks +6 days of gestation [35]. These guidelines state that for women presenting with suspected preeclampsia at $<35$ weeks of gestation (base case), total costs were $£ 6,456$ when using the Elecsys sFlt-1/PlGF ratio test, compared with $£ 8,945$ for the standard of care, yielding cost savings of $£ 2,488$ per patient. However, it must be considered that NICE guidelines are based on the UK healthcare system and thus cannot directly inform or be used as a comparator for clinical practice in Japan. A cutoff value of $\leq 38$ has a high NPV of 99.3\% for ruling out the onset of preeclampsia within 1 week [17, 22]. Analysis of the economic impact in the UK indicated that hospitalizations of women with suspected preeclampsia were reduced by $56 \%$, resulting in net savings of $£ 344$ per patient for the healthcare provider [28]. The substantial cost saving results per patient were replicated in Italy (€670) [25], Germany (€361) [27], Brazil (R \$185-636) [24], Switzerland (€346) [26], and the US $(\$ 1,215)$ [29]. Taken together, these studies show that the sFlt-1/PIGF ratio test yields cost savings for different populations/geographical areas and from the perspective of a range of healthcare providers with different procedures and financial models.

A strength of the present analysis is that the economic model used real-world, patient-level data collected from a large observational study (PROGNOSIS Asia) [15]. From these data, we were able to extract data from the Japanese cohort. Thus, our results are highly likely to be representative of current clinical practice for managing preeclampsia in Japan.

A further strength of the present analysis is that our modeling included the cost of neonatal care (i.e., NICU admission and corticosteroid treatment) for neonates with RDS born to mothers with preeclampsia. Previous research has suggested that, in the context of preeclampsia, the cost burden for neonatal care may exceed that for maternal care in the first 12 months after delivery [9]. Furthermore, research has shown that the use of antenatal corticosteroids may reduce the costs of medical services and the length of hospitalization for preterm infants [36]. The inclusion of these costs in our model ensures that the cost-saving results of our study provide an accurate representation of the burden of preeclampsia on the Japanese healthcare system. A range of sensitivity analyses were performed to check the 
robustness of our results to changes in key model parameters.

This study has limitations. It is conceded that a randomized interventional study on the actual economic impact of using the sFlt-1/PIGF ratio to rule out preeclampsia could further strengthen our findings by quantifying the value of the test more accurately in routine clinical practice. Moreover, incorporating additional data on delivery and birth outcome parameters (e.g., length of pregnancy, cesarean section rate, birth weight) into the test and no-test scenarios could further inform this model; however, these data could not be included in the present study due to a lack of available data. Furthermore, this model assumes that all women treated with high-intensity management are prescribed oral corticosteroids to reduce the risk of complications; however, Japanese guidelines recommend that corticosteroids are administered by intramuscular injection rather than administered orally [34]. Therefore, the cost savings in real-world practice may differ slightly from those presented in the present model.

\section{Conclusions}

Our economic analysis demonstrates that implementing the sFlt-1/PlGF ratio test for the management of women with suspected preeclampsia in Japan may reduce unnecessary hospitalizations. Furthermore, reducing unnecessary hospitalizations is associated with cost savings for Japanese healthcare providers.

Acknowledgements Third-party medical writing assistance, under the direction of the authors, was provided by Chloe Fletcher, MSc (Ashfield MedComms, Macclesfield, UK), and was funded by Roche Diagnostics International Ltd. (Rotkreuz, Switzerland). COBAS, COBAS E and ELECSYS are trademarks of Roche.

Funding This study was funded by Roche Diagnostics International Ltd. (Rotkreuz, Switzerland).

Author contributions T Kikuchi, N Taguchi, C Wolf: study design and concept development, analysis and interpretation of data. A Ohkuchi, H Masuyama, T Yamamoto, S Saito: collection and interpretation of data. All authors drafted the manuscript and approved the final version for submission.

\section{Compliance with ethical standards}

Conflict of interest TK and NT are full-time employees of Roche Diagnostics K.K. CW is a full-time employee of Roche Diagnostics International Ltd. AO, TY and SS received other fees from Roche Diagnostics during the conduct of the study. HM has no conflicts of interest to disclose.

Publisher's note Springer Nature remains neutral with regard to jurisdictional claims in published maps and institutional affiliations.
Open Access This article is licensed under a Creative Commons Attribution 4.0 International License, which permits use, sharing, adaptation, distribution and reproduction in any medium or format, as long as you give appropriate credit to the original author(s) and the source, provide a link to the Creative Commons license, and indicate if changes were made. The images or other third party material in this article are included in the article's Creative Commons license, unless indicated otherwise in a credit line to the material. If material is not included in the article's Creative Commons license and your intended use is not permitted by statutory regulation or exceeds the permitted use, you will need to obtain permission directly from the copyright holder. To view a copy of this license, visit http://creativecommons. org/licenses/by/4.0/.

\section{References}

1. Tranquilli AL, Dekker G, Magee L, Roberts J, Sibai BM, Steyn $\mathrm{W}$, et al. The classification, diagnosis and management of the hypertensive disorders of pregnancy: a revised statement from the ISSHP. Pregnancy Hypertens. 2014;4:97-104.

2. Abalos E, Cuesta C, Carroli G, Qureshi Z, Widmer M, Vogel J, et al. Pre-eclampsia, eclampsia and adverse maternal and perinatal outcomes: a secondary analysis of the World Health Organization Multicountry Survey on Maternal and Newborn Health. BJOG. 2014;121:14-24.

3. Duley L. The Global Impact of Pre-eclampsia and Eclampsia. Semin Perinatol. 2009;33:130-7.

4. Shiozaki A, Matsuda Y, Satoh S, Saito S. Comparison of risk factors for gestational hypertension and preeclampsia in Japanese singleton pregnancies. J Obstet Gynaecol Res. 2013;39: 492-9.

5. Liu A, Wen SW, Bottomley J, Walker MC, Smith G. Utilization of health care services of pregnant women complicated by preeclampsia in Ontario. Hypertens Pregnancy. 2009;28:76-84.

6. Lo JO, Mission JF, Caughey AB. Hypertensive disease of pregnancy and maternal mortality. Curr Opin Obstet Gynecol. 2013;25:124-32.

7. Kanayama N, Inori J, Ishibashi-Ueda H, Takeuchi M, Nakayama M, Kimura S, et al. Maternal death analysis from the Japanese autopsy registry for recent 16 years: significance of amniotic fluid embolism. J Obstet Gynaecol Res. 2011;37:58-63.

8. Delahaije DH, Smits LJ, van Kuijk SM, Peeters LL, Duvekot JJ, Ganzevoort W, et al. Care-as-usual provided to formerly preeclamptic women in the Netherlands in the next pregnancy: health care consumption, costs and maternal and child outcome. Eur J Obstet Gynecol Reprod Biol. 2014;179:240-5.

9. Stevens W, Shih T, Incerti D, Ton TGN, Lee HC, Peneva D, et al. Short-term costs of preeclampsia to the United States health care system. Am J Obstet Gynecol. 2017;217:237-248.e216.

10. Minakami H, Maeda T, Fujii T, Hamada H, Iitsuka Y, Itakura A, et al. Guidelines for obstetrical practice in Japan: Japan Society of Obstetrics and Gynecology (JSOG) and Japan Association of Obstetricians and Gynecologists (JAOG) 2014 edition. J Obstet Gynaecol Res. 2014;40:1469-99.

11. Levine RJ, Lam C, Qian C, Yu KF, Maynard SE, Sachs BP, et al. Soluble endoglin and other circulating antiangiogenic factors in preeclampsia. N Engl J Med. 2006;355:992-1005.

12. Levine RJ, Maynard SE, Qian C, Lim K-H, England LJ, Yu KF, et al. Circulating Angiogenic Factors and the Risk of Preeclampsia. N Engl J Med. 2004;350:672-83.

13. Verlohren S, Galindo A, Schlembach D, Zeisler H, Herraiz I, Moertl MG, et al. An automated method for the determination of the sFlt-1/PIGF ratio in the assessment of preeclampsia. Am J Obstet Gynecol. 2010;202:161.e161-161.e111. 
14. Verlohren S, Herraiz I, Lapaire O, Schlembach D, Moertl M, Zeisler $\mathrm{H}$, et al. The sFlt-1/PlGF ratio in different types of hypertensive pregnancy disorders and its prognostic potential in preeclamptic patients. Am J Obstet Gynecol. 2012;206:58. e51-58.

15. Bian X, Biswas A, Huang X, Lee KJ, Li TK-T, Masuyama H, et al. Short-Term Prediction of Adverse Outcomes Using the sFlt1 (Soluble fms-Like Tyrosine Kinase 1)/PlGF (Placental Growth Factor) Ratio in Asian Women With Suspected Preeclampsia. Hypertension. 2019;74:164-72.

16. Cerdeira AS, O'Sullivan J, Ohuma EO, Harrington D, Szafranski P, Black R, et al. Randomized Interventional Study on Prediction of Preeclampsia/Eclampsia in Women With Suspected Preeclampsia. Hypertension. 2019;74:983-90.

17. Hund M, Allegranza D, Schoedl M, Dilba P, VerhagenKamerbeek W, Stepan H. Multicenter prospective clinical study to evaluate the prediction of short-term outcome in pregnant women with suspected preeclampsia (PROGNOSIS): study protocol. BMC Pregnancy Childbirth. 2014;14:324.

18. Ohkuchi A, Hirashima C, Suzuki H, Takahashi K, Yoshida M, Matsubara S, et al. Evaluation of a new and automated electrochemiluminescence immunoassay for plasma sFlt-1 and PlGF levels in women with preeclampsia. Hypertension Res. 2010;33: 422-7.

19. Perales A, Delgado JL, de la Calle M, García-Hernández JA, Escudero AI, Campillos JM, et al. sFlt-1/PlGF for prediction of early-onset pre-eclampsia: STEPS (Study of Early Pre-eclampsia in Spain). Ultrasound Obstet Gynecol. 2017;50:373-82.

20. Stepan H, Hund M, Andraczek T. Combining Biomarkers to Predict Pregnancy Complications and Redefine Preeclampsia: the Angiogenic-Placental Syndrome. Hypertension. 2020;75:918-26.

21. Verlohren S, Herraiz I, Lapaire O, Schlembach D, Zeisler H, Calda $\mathrm{P}$, et al. New gestational phase-specific cutoff values for the use of the soluble fms-like tyrosine kinase-1/placental growth factor ratio as a diagnostic test for preeclampsia. Hypertension. 2014;63:346-52.

22. Zeisler H, Llurba E, Chantraine F, Vatish M, Staff AC, Sennström M, et al. Predictive Value of the sFlt-1:PlGF Ratio in Women with Suspected Preeclampsia. N Engl J Med. 2016;374:13-22.

23. Zeisler H, Llurba E, Chantraine FJ, Vatish M, Staff AC, Sennström M, et al. Soluble fms-like tyrosine kinase- 1 to placental growth factor ratio: ruling out pre-eclampsia for up to 4 weeks and value of retesting. Ultrasound Obstet Gynecol. 2019;53:367-75.

24. Figueira SF, Wolf C, D'Innocenzo M, de Carvalho JPV, Barbosa MG, Zlotnik E, et al. Economic evaluation of sFlt-1/PlGF ratio test in pre-eclampsia prediction and diagnosis in two Brazilian hospitals. Pregnancy Hypertens. 2018;13:30-36.

25. Frusca T, Gervasi M-T, Paolini D, Dionisi M, Ferre F, Cetin I. Budget impact analysis of sFlt-1/PIGF ratio as prediction test in Italian women with suspected preeclampsia. J Matern Fetal Neonatal Med. 2017;30:2166-73.

26. Hodel M, Blank PR, Marty P, Lapaire O. sFlt-1/PlGF Ratio as a Predictive Marker in Women with Suspected Preeclampsia: an
Economic Evaluation from a Swiss Perspective. Dis Markers. 2019;2019:4096847.

27. Schlembach D, Hund M, Schroer A, Wolf C. Economic assessment of the use of the sFlt-1/PIGF ratio test to predict preeclampsia in Germany. BMC Health Serv Res. 2018;18:603-603.

28. Vatish M, Strunz-McKendry T, Hund M, Allegranza D, Wolf C, Smare C. sFlt-1/PIGF ratio test for pre-eclampsia: an economic assessment for the UK. Ultrasound Obstet Gynecol. 2016;48:765-71.

29. Schnettler WT, Dukhovny D, Wenger J, Salahuddin S, Ralston SJ, Rana S. Cost and resource implications with serum angiogenic factor estimation in the triage of pre-eclampsia. BJOG. 2013;120: 1224-32.

30. Schlembach D, Hund M, Wolf C, Vatish M. Diagnostic utility of angiogenic biomarkers in pregnant women with suspected preeclampsia: a health economics review. Pregnancy Hypertens. 2019;17:28-35.

31. Ohkuchi A, Saito S, Yamamoto T, Minakami H, Masuyama H, Kumasawa K, et al. Short-term prediction of preeclampsia using the sFlt-1/PIGF ratio: a subanalysis of pregnant Japanese women from the PROGNOSIS Asia study. Hypertens Res. 2021 [In press]. https://doi.org/10.1038/s41440-021-00629-x.

32. Neonatal Research Network of Japan. Neonatal Research Network of Japan Database - Analysis results on infants born in 2016. https://plaza.umin.ac.jp/nrndata/reports/nrn1_2016.pdf. Accessed 18 June 2020.

33. Klein E, Schlembach D, Ramoni A, Langer E, Bahlmann F, Grill $\mathrm{S}$, et al. Influence of the sFlt-1/PIGF Ratio on Clinical Decision-Making in Women with Suspected Preeclampsia. PLoS ONE 2016;11:e0156013. https://doi.org/10.1371/journal. pone. 0156013.

34. Takagi K, M Y, Nakamoto O, S S, H S, Seki H, et al. A review of best practice guide 2015 for care and treatment of hypertension in pregnancy. Hypertens Res Pregnancy. 2015;3:65-102.

35. National Institute for Healthcare Excellence (NICE). PlGF-based testing to help diagnose suspected pre-eclampsia (Triage PIGF test, Elecsys immunoassay sFlt-1/PIGF ratio, DELFIA Xpress PlGF 1-2-3 test, and BRAHMS sFlt-1 Kryptor/BRAHMS PlGF plus Kryptor PE ratio). https://www.nice.org.uk/guidance/dg23/ chapter/1-Recommendations. Accessed 23 April 2020.

36. Ogata JF, Fonseca MC, Miyoshi MH, Almeida MF, Guinsburg R. Costs of hospitalization in preterm infants: impact of antenatal steroid therapy. J Pediatr (Rio J). 2016;92:24-31.

37. Ministry of Health LaW. List of NHI drug price listed items and information on generic drugs, 2019. https://www.mhlw.go.jp/ topics/2019/08/tp20190819-01.html. Accessed 18 June 2020.

38. Ministry of Health LaW. Report of the survey 'Discharge Patient Survey' concerning impact assessment of the 2017 DPC introduction. https://www.mhlw.go.jp/stf/shingi2/0000196043_00001. html. Accessed 18 June 2020.

39. Ministry of Health LaW. Survey of Medical Care Activities in Public Health Insurance, 2016. https://www.mhlw.go.jp/english/ database/db-hss/dl/smcaphi_y_2016.pdf. Accessed 14 May 2020. 